\title{
Etude de la circulation à l'intérieur du caisson Jarlan
}

\author{
E.H. Tabet Aoul, Maître de conférence associé-Université du Havre \\ M. Bélorgey, Professeur Université de Caen
}

\section{Résumé}

Le présent papier traite de la circulation de l'eau à l'intérieur du caisson à paroi perforée, type Jarlan, et de l'évolution de la réflexion en fonction du rapport de la largeur de la chambre sur la longueur d'onde ainsi que du rapport des profondeurs d'eau à l'intérieur et à l'extérieur du caisson. Les résultats montrent que l'optimum est obtenu pour un rapport de l'ordre de 0,16 à 0,18 .

Une présentation des mesures de champs de vitesse par VDL à l'intérieur du caisson ainsi que des mesures des fluctuations des niveaux de la surface libre de part et d'autre de la paroi perforée est faite. Une approche du bilan énergétique montre qu'il existe en fait 4 facteurs contribuant à la dissipation de l'énergie.

Il donne ainsi des indications pouvant contribuer à une meilleure compréhension du mode de fonctionnement hydraulique de ce type d'ouvrage.

\section{Introduction}

Le caisson Jarlan comporte entre la paroi amont perforée et la paroi avale pleine, un espace participant à la dissipation de l'énergie transmise. Son fonctionnement hydraulique dépend des paramètres géométriques du caisson ainsi que des caractéristiques de la houle incidente.

Le problème est de rechercher les relations liant la réflexion partielle de l'onde incidente sur l'obstacle créé par le caisson, à ces différents paramètres.

\section{Analyse de la réflexion}

Les études ont porté sur l'influence de la géométrie du caisson et des caractéristiques de la houle incidente sur la dissipation de l'énergie à l'intérieur de l'ouvrage.

Pour chaque période de houle générée, la longueur d'onde correspondante est donnée au droit du caisson avec une profondeur d'eau égale à $40 \mathrm{~cm}$ soit $10 \mathrm{~m}$ en nature. La figure 1 montre que le coefficient de réflexion varie selon une courbe en cloche renversée avec le minimum atteint pour une fréquence de l'ordre de 0.55 $\mathrm{Hz}(0.11 \mathrm{~Hz}$ en nature) et un rapport $\mathrm{B} / \mathrm{L}=0.18$. Ce résultat confirme ceux publiés par W. Allsop (1994) et ceux de Tanimoto (1995).

Il apparaît ainsi que la réflexion minimale et donc l'efficacité maximale du caisson perforé est à situer autour de $\mathrm{B} / \mathrm{L}=0,15$ à 0,20 .

Par ailleurs et pour étudier l'influence de la profondeur d'eau à l'intérieur du caisson, une série d'essais a été faite en rehaussant le niveau du fond du caisson de 
manière à avoir le rapport $d / h=0.625$ au lieu de $d / h=1$ tel que conçu initialement.

$\mathrm{d}$ : profondeur d'eau à l'intérieur du caisson

h: " " devant le caisson

B: largeur du caisson

$\mathrm{Hi}, \mathrm{L}$ et $\mathrm{T}$ : caractéristiques de la houle incidente au large du caisson

La comparaison entre les 2 courbes de la figure ci dessous montre que plus la profondeur d'eau dans le caisson est faible plus le coefficient de réflexion $\mathrm{Cr}$ correspondant à la même énergie incidente augmente.

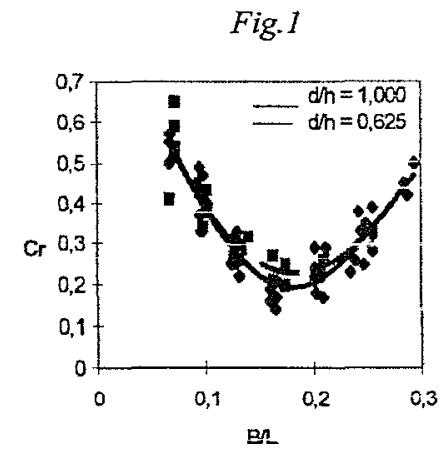

Ceci est mis en évidence en particulier pour les valeurs de $B / L$ où la réflexion est minimale, c'est-à-dire lorsque la dissipation de l'énergie dans le caisson est maximale.

$\mathrm{Ce}$ même phénomène est montré dans les résultats théoriques donnés par Tanimoto et al (1982) où Cr est calculé en fonction de la porosité $\varepsilon$ et du rapport $\mathrm{q}=\mathrm{d} / \mathrm{h}$. Il apparaît, en particulier, que pour les faibles poresités inférieures à 0.25 , le coefficient de réflexion augmente lorsque $\mathrm{d} / \mathrm{h}$ diminue; de même que les minima des courbes $\mathrm{Cr}=\mathrm{f}(\varepsilon)$ augmentent lorsque $\mathrm{d} / \mathrm{h}$ diminue.

A noter néanmoins que pour $\mathrm{B} / \mathrm{L}<0.1 \mathrm{c}$-à-d pour les grandes longueurs d'ondes (dans notre cas pour $\mathrm{L}>135 \mathrm{~m}$ en nature ou $\mathrm{T}>14 \mathrm{~s}$ ) et pour $\mathrm{B} / \mathrm{L}>0.33(\mathrm{~L}<40$ $\mathrm{m}$ ou $\mathrm{T}<5.3 \mathrm{~s}$ ) le coefficient de réflexion n'est affecté ni par la porosité ni par la profondeur d'eau dans le caisson.

Par contre son effet est significatif dans l'intervalle $0.10<\mathrm{B} / \mathrm{L}<0.33$, là où la dissipation d'énergie est maximum et donc là où la fréquence d'excitation de la houle est proche de la fréquence propre du caisson.

\section{La circulation autour du caisson}

Quatre campagnes de mesure de vitesse par vélocimétrie Laser à l'intérieur du caisson ont été réalisées.

Les houles générées pour chacune des 4 campagnes ont les caractéristiques suivantes: 


\begin{tabular}{|l|l|l|l|l|}
\hline & $T_{/ s}$ & $L_{/ m}$ & $H i_{/ c m}$ & $C r$ \\
\hline lère campagne & 1,30 & 2,19 & 10,6 & 0,28 \\
\hline 2ème camp. & 1,54 & 2,68 & 8,2 & 0,25 \\
\hline 3ème camp. & 1,82 & 3,31 & 6,2 & 0,17 \\
\hline 4ème camp. & 2,94 & 5,65 & 4,1 & 0,35 \\
\hline
\end{tabular}

Le système enregistre les données des 2 composantes de la vitesse dans le plan de propagation de la houle. La corrélation du point de mesure de la vitesse avec la position de la vague est assurée grâce à une sonde résistive placée devant l'ouvrage. Cette sonde donne l'évolution du niveau de la surface libre. Elle est reliée à un 'trigger" conçu pour déclencher une impulsion électrique à intervalle régulier. Cet intervalle est calé sur la fréquence de la houle enregistrée par la sonde. La durée d'acquisition est programmée de manière à donner un enregistrement sur un nombre de vagues suffisamment grand pour être représentatif du phénomène à étudier. Dans le cas présent et compte tenu du type de houle considérée ( $\mathrm{T}$ variant de 1,3 à $3 \mathrm{~s}$ en modèle), la durée d'échantillonnage retenue a été de $30 \mathrm{~s}$, correspondant à un nombre de vagues allant de 10 à 25 vagues.

Chaque enregistrement est soumis à un traitement statistique. Le programme retient un nombre entier de vagues grâce à la présence de 'bips"' donnés par le trigger. Chaque vague est ensuite découpée en un grand nombre d'intervalles appelés phases (50 dans notre cas), dont sont tirées les composantes Ui et Vi de la vitesse, considérées comme instantanées $(\Delta t=T / 50=0,02$ à $0,06 \mathrm{~s}$ pour $1 \mathrm{~s}<\mathrm{T}<3 \mathrm{~s}$ ). Les vagues retenues sont ensuite superposées et un programme de lissage (Colmard C. -1996) donne la vague représentative de l'échantillon de houle générée dans le canal. On peut ainsi faire ressortir pour chaque vague représentative, les composantes horizontales $\mathrm{Ui}$ et verticales $\mathrm{Vi}$ de la vitesse instantanée en tout point situé dans le plan de propagation de la houle.

Les composantes de la vitesse peuvent être aussi moyennées sur une période telles que:

$$
\begin{aligned}
\text { Umoy } & =\sum(\mathrm{Ui} / \mathrm{N}) \\
\mathrm{Vmoy} & =\sum(\mathrm{Vi} / \mathrm{N}) \\
\mathrm{N} & : \text { nombre de phases }
\end{aligned}
$$

C'est ainsi qu'ont pu être calculées pour les 4 campagnes citées ci-dessus, les champs moyens des vitesses des particules fluides à l'intérieur du caisson.

Les composantes horizontales et verticales de la vitesses ont été mesurées point par point selon des maillages de $1 \mathrm{cmx} 1 \mathrm{~cm}$ pour la campagne $3,2 \mathrm{x}$

et $6 \times 6$ pour la 2 . Les points enregistrés sont distribués dans

$54 \mathrm{~cm} \times 34 \mathrm{~cm}$ dans un plan transversal au caisson passant par $\mathrm{l}^{\text {: }}$ perforations se trouvant sur une même verticale.

Les figures 2, 3 et 4 représentent les champs de vitesses moyenne: caisson pour 3 types de périodes de la houle incidente. 

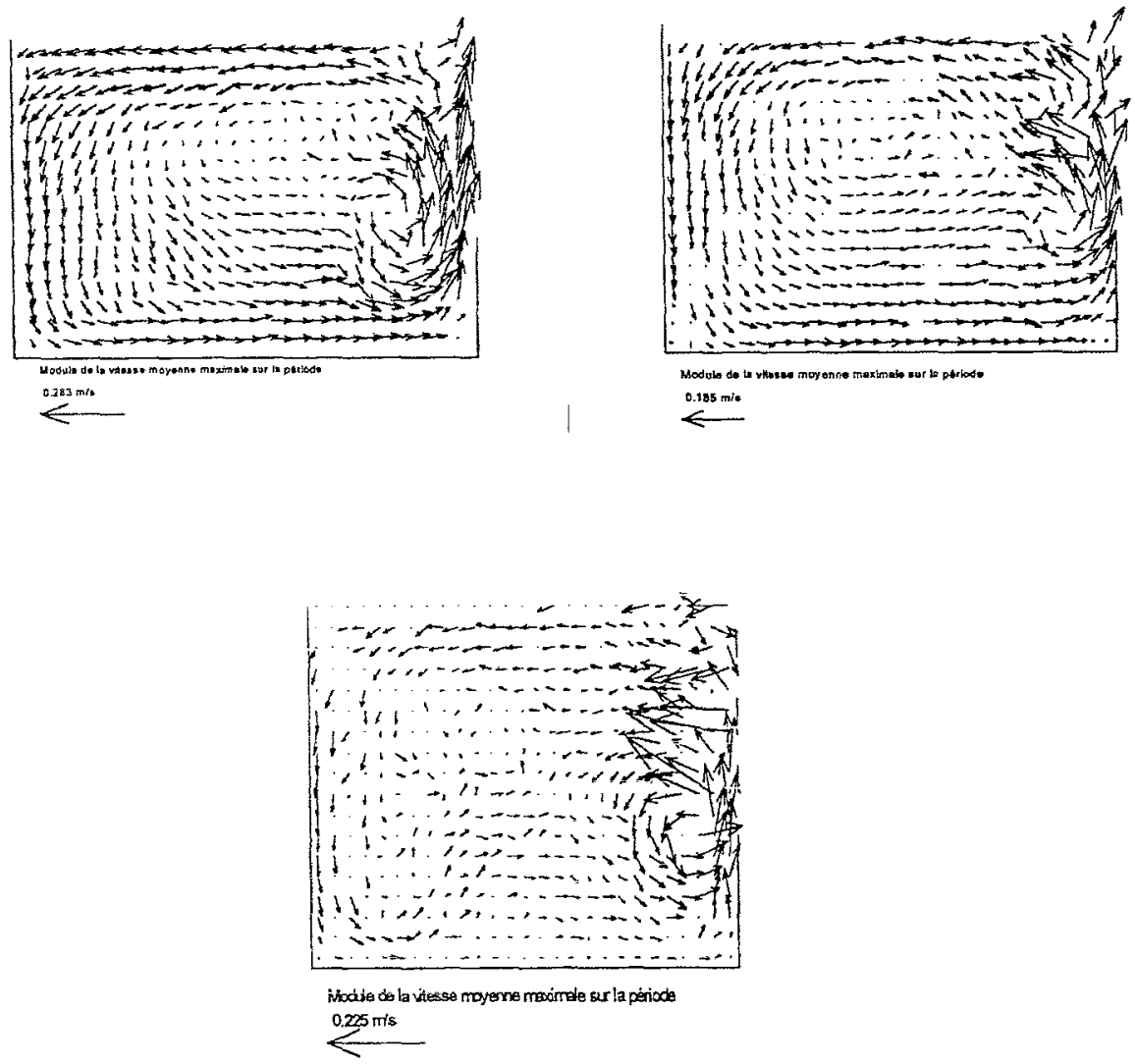

Fig.4 Période $=2.94$ s

Il a pu être mis en évidence un mouvement giratoire d'ensemble dans la chambre dans le sens contraire des aiguilles d'une montre. La direction est d'abord plutôt ascendante vers la surface libre, dans le sens paroi perforée - paroi pleine dans les tranches supérieures du fluide puis descendante lelong de la paroi opaque et enfin retour près du fond vers la paroi perforée.

Ce mouvement moyen, assez perturbé dans le cas de la plus grande période $(\mathrm{B} / \mathrm{L}=0,10)$, croît avec la fréquence de la houle. Il devient particulièrement important pour $B / L=0,25$ où les vitesses y sont élevées, pouvant atteindre 15 à 20 $\mathrm{cm} / \mathrm{s}$ prés de la paroi perforée (prés d' $1 \mathrm{~m} / \mathrm{s}$ en nature).

\section{Bilan de l'énergie dissipée par la présence du caisson}

\section{III.1 Calcul de l'énergie incidente}

Le calcul de l'énergie incidente par mètre linéaire de largeur du caisson $\mathrm{Ei} / \mathrm{ml}$, peut être fait en considérant l'énergie traversant un plan vertical pendant une période sous la forme de:

$$
E i_{/ m l}=\rho g H i^{2} L(1+4 \pi d / L / \sinh (4 \pi d / L)) / 16
$$


d: profondeur d'eau dans le canal prise égale à $40 \mathrm{~cm}$

L'énergie dissipée dans le caisson Ed par mètre linéaire, peut être aussi évaluée comme étant égale à la différence entre l'énergie incidente et l'énergie réfléchie, soit:

$$
E d_{/ m l}=E i\left(1-C r^{2}\right)
$$

Le calcul donne en particulier pour:

$$
\begin{aligned}
& \mathrm{T}=1,30 \mathrm{~s} \quad \mathrm{Ei}_{\mathrm{ml}}=22,14 \mathrm{~J} / \mathrm{m} \\
& \mathrm{T}=1,54 \mathrm{~s} \quad=17,56 \mathrm{~J} / \mathrm{m} \\
& \mathrm{T}=1,82 \mathrm{~s} \quad=13,25 \mathrm{~J} / \mathrm{m} \\
& \mathrm{T}=2,94 \mathrm{~s} \quad=10,94 \mathrm{~J} / \mathrm{m} \\
& \mathrm{Ed}_{/ \mathrm{ml}}=20,40 \mathrm{~J} / \mathrm{m} \\
& =16,46 \mathrm{~J} / \mathrm{m} \\
& =12,87 \mathrm{~J} / \mathrm{m} \\
& =9,60 \mathrm{~J} / \mathrm{m}
\end{aligned}
$$

\section{III.2 Calcul de la circulation autour du caisson.}

La circulation de la vitesse lelong d'un contour tracé dans le caisson s'écrit:

$$
\Gamma=f_{c} V \cdot d l
$$

Le calcul a été fait en considérant un rectangle de fluide dans le caisson dont les sommets sont les points $M_{1}(1,1), M_{2}(1,33), M_{3}(53,33), M_{4}(53,1)$

Pour tenter une estimation de la répartition de l'énergie dissipée dans le caisson, il a été procédé au calcul de la circulation générale $\Gamma$, mise en évidence dans le caisson.

$\Gamma$ est calculée en discrétisant l'intégrale avec dl de composantes $\Delta \mathrm{x}_{\mathrm{i}}, \Delta \mathrm{z}_{\mathrm{i}}$ et $\mathrm{V}$ de composantes $u_{\mathrm{i}}, \mathrm{w}_{\mathrm{i}}$

$$
\Gamma=\Sigma\left(u_{i} \Delta x_{i}+w_{i} \Delta z_{i}\right)
$$

On peut écrire aussi grâce à la formule de Stokes que la circulation des vitesses lelong de ce contour fermé est égale au flux du vecteur tourbillon à travers ce contour.

Nous nous sommes proposés de calculer ainsi le travail $W$ en le considérant comme étant égal au produit de cette circulation par la masse d'eau mise en mouvement à l'intérieur du caisson; ceci rapporté à la période de la houle incidente.

$$
W_{/ m l}=\rho \cdot S \cdot \Gamma / T
$$

avec $\rho S$ : masse d'eau par ml de caisson, soumise à cette circulation

$\mathrm{T}$ : période de la houle incidente

Le calcul a donné pour:

$$
\begin{array}{lll}
\mathrm{T}=1,30 \mathrm{~s} & \Gamma=9,43 \times 10^{-2} \mathrm{~m}^{2} / \mathrm{s} & \mathrm{W}_{/ \mathrm{ml}}=12,7 \mathrm{~J} / \mathrm{m} \\
\mathrm{T}=1,54 \mathrm{~s} & \Gamma=5,95 \times 10^{-2} \mathrm{~m}^{2} / \mathrm{s} & \mathrm{W}_{/ \mathrm{ml}}=6,76 \mathrm{~J} / \mathrm{m} \\
\mathrm{T}=1,82 \mathrm{~s} & \Gamma=4,34 \times 10^{-2} \mathrm{~m}^{2} / \mathrm{s} & \mathrm{W}_{/ \mathrm{ml}}=4,17 \mathrm{~J} / \mathrm{m} \\
\mathrm{T}=2,94 \mathrm{~s} & \Gamma=4,0 \times 10^{-2} \mathrm{~m}^{2} / \mathrm{s} & \mathrm{W}_{/ \mathrm{ml}}=2,64 \mathrm{~J} / \mathrm{m}
\end{array}
$$




\section{III.3 Calcul de l'énergie dissipée au passage des perforations}

Cette énergie dissipée peut être calculée comme une perte de charge due à la contraction de l'écoulement au passage des trous.

On peut considérer que le modèle physique a été conçu de manière à ce que la saturation du régime turbulent soit atteinte; c-à-d que la turbulence devient prépondérante par rapport aux forces visqueuses.

Comme dans le cas présent, les perforations ont des diamètres de l'ordre de leur longueur, l'écoulement peut être considéré comme traversant un ouvrage court. Dans cette zone le coefficient de perte de charge est constant. Nous le prendrons égal à $\mathrm{k}=0,6$ comme le préconise Natale (1995)

$$
\Delta H=0,6 \mathrm{Um}^{2} / 2 g
$$

Um est donnée cí dessous comme la vitesse moyenne enregistrée à la sortie de chacun des trous situés sur une même verticale (à $15 \mathrm{~cm}$ du fond pour le trou $\mathrm{N}^{2} 1$, les autres étant distants de $6,7 \mathrm{~cm}$ entre axes).

\begin{tabular}{|l|l|l|l|l|}
\hline & Trou $N^{\circ} 1$ & Trou $N^{\circ} 2$ & Trou $N^{\circ} 3$ & Trou $N^{\circ} 4$ \\
\hline$T=1,30 \mathrm{~s}$ & $5,5 \mathrm{~cm} / \mathrm{s}$ & $7 \mathrm{~cm} / \mathrm{s}$ & $4 \mathrm{~cm} / \mathrm{s}$ & $5 \mathrm{~cm} / \mathrm{s}$ \\
\hline$T=1,82 \mathrm{~s}$ & $4,2 \mathrm{~cm} / \mathrm{s}$ & $18,3 \mathrm{~cm} / \mathrm{s}$ & $12,4 \mathrm{~cm} / \mathrm{s}$ & $9,3 \mathrm{~cm} / \mathrm{s}$ \\
\hline$T=2,94 \mathrm{~s}$ & $4,2 \mathrm{~cm} / \mathrm{s}$ & $18,3 \mathrm{~cm} / \mathrm{s}$ & $12,3 \mathrm{~cm} / \mathrm{s}$ & $3,4 \mathrm{~cm} / \mathrm{s}$ \\
\hline
\end{tabular}

Nombre de trous utilisés: $N=4 \times 15$

De ces résultats l'on a pu faire un bilan énergétique en supposant que cette énergie perdue par perte de charge aurait pu être stockée sous forme d'énergie potentielle telle que: $\quad E_{p d c / m l}=\rho . g \cdot \Delta H^{2} \cdot B$

Le calcul donne ainsi:

$\begin{array}{lll}\text { pour } T=1,30 \mathrm{~s} & \Delta \mathrm{H}_{1}=0,55 \mathrm{~cm} & \mathrm{E}_{\mathrm{pdc}}=0,16 \mathrm{~J} / \mathrm{ml} \\ \text { pour } \mathrm{T}=1,54 \mathrm{~s} & \Delta \mathrm{H}_{2}=0,58 \mathrm{~cm} & \mathrm{E}_{\mathrm{pdc}}=0,18 \mathrm{~J} / \mathrm{ml} \\ \text { pour } \mathrm{T}=1,82 \mathrm{~s} & \Delta \mathrm{H}_{3}=2,72 \mathrm{~cm} & \mathrm{E}_{\mathrm{pdc}}=3,92 \mathrm{~J} / \mathrm{ml} \\ \text { pour } \mathrm{T}=2,94 \mathrm{~s} & \Delta \mathrm{H}_{4}=2,36 \mathrm{~cm} & \mathrm{E}_{\mathrm{pdc}}=2,95 \mathrm{~J} / \mathrm{ml}\end{array}$

\section{III.4 Calcul de l'énergie potentielle récupérée au dessus du niveau moyen}

L'enregistrement des fluctuations du niveau de la surface libre a montré que les niveaux moyens à l'intérieur et à l'extérieur du caisson varient en fonction des caractéristiques de la houle générée.

La figure 5 a été construite à partir de plusieurs essais réalisés en faisant varier les hauteurs et périodes de la houle incidente. Il apparaît ainsi que le niveau moyen à l'intérieur est supérieur au niveau à l'extérieur pour toutes les valeurs de $\mathrm{B} / \mathrm{L}<0,25$. Par contre cette différence change de signe et croit rapidement pour les hautes fréquences de la houle. 


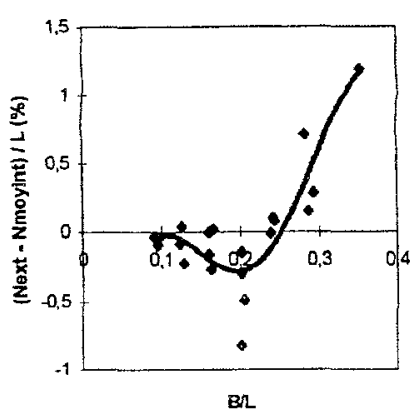

Fig. 5

La houle générée étant monochromatique, elle ne doit pas produire de surélévation du niveau moyen; ces variations du niveau moyen peuvent donc être considérées comme de l'énergie potentielle stockée. Nous nous sommes proposés de la calculer et de tracer les courbes correspondantes, en considérant l'énergie potentielle $E_{\text {pint }}$ stockée à l'intérieur du caisson :

$$
E_{\text {pint } / m l}=\rho g N_{\text {int }}^{2} B
$$

$\mathrm{N}_{\text {int: }}$ surélévation moyenne du niveau moyen à l'intérieur du caisson

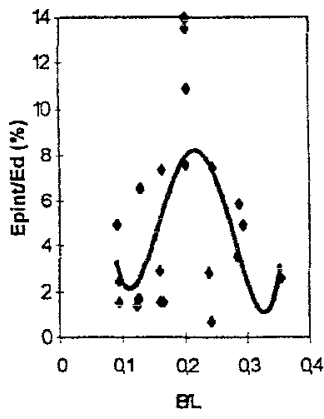

Fig. 6

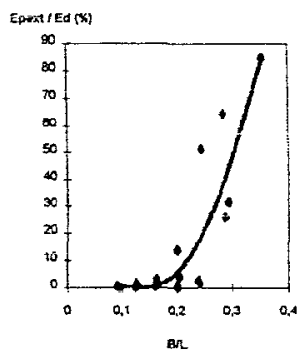

Fig. 7

Des résultats de la figure 6 , on déduit pour chacune des campagnes citées précédemment, les parts $\mathrm{E}_{\text {pint }}$ de l'énergie dissipée à l'intérieur du caisson et récupérée en énergie potentielle.

Un calcul similaire a été fait pour la surélévation du niveau devant la paroi perforée, qui ne peut pas être une surélévation classique due à la houle incidente (monochromatique). Les observations faites en modèie ont montré que cette surélévation s'étendait sur environ $1 / 4$ de la longueur d'onde. La figure 7 montre que l'énergie potentielle produite rapportée à l'énergie totale dissipée est faible pour le cas de $B / L<0,25$, mais devient relativement importante pour les hautes fréquences de la houle

\section{$\underline{\text { IV. Conclusion }}$}

La synthèse de cet ensemble de résultats donne un aperçu global de la répartition de l'énergie dissipée par mètre linéaire de caisson. 
Le tableau ci-après résume cette répartition:

\begin{tabular}{|c|c|c|c|c|c|c|c|c|}
\hline$T_{/ s}$ & $B / L$ & $E_{i \mathrm{~J} / m l}$ & $E_{d \mathrm{~J} / m l}$ & $W / E_{d}$ & $E_{p d d} \boldsymbol{E}_{d}$ & $\boldsymbol{E}_{\text {pint }} \boldsymbol{E}_{d}$ & $\boldsymbol{E}_{\text {pexd }}$ & $\Sigma / \boldsymbol{E}_{d}$ \\
\hline 1,30 & 0,24 & 22,14 & 20,40 & $62,3 \%$ & $0,8 \%$ & $2 \%$ & $10 \%$ & $75,1 \%$ \\
\hline 1,54 & 0,20 & 17,56 & 16,46 & $41,0 \%$ & $1 \%$ & $14 \%$ & $5 \%$ & $61,0 \%$ \\
\hline 1,82 & 0,16 & 13,25 & 12,87 & $32,4 \%$ & $30,4 \%$ & $6 \%$ & $2 \%$ & $70,8 \%$ \\
\hline 2,94 & 0,10 & 10,94 & 9,60 & $27,5 \%$ & $30,7 \%$ & $3 \%$ & $0 \%$ & $70,8 \%$ \\
\hline
\end{tabular}

Il apparait ainsi que la partie dissipée par la circulation à l'intérieur du caisson croît avec la fréquence de la houle de l'ordre de $1 / 4$ à $3 / 4$ de l'énergie totale dissipée. La perte de charge par contre n'est significative que pour les houles de périodes moyennes ou longues alors que l'énergie potentielle stockée à l'intérieur du caisson est importante dans le cas où le coefficient de réflexion est optimal. Enfin l'énergie potentielle stockée devant la paroi perforée, négligeable pour les houles de faibles fréquences, devient importante et croît rapidement avec les grandes fréquences.

Ce bilan énergétique dont la somme varie entre $60 \%$ et prés de $75 \%$ de l'énergie totale dissipée, donne des indications assez claire sur le mode de fonctionnement hydraulique du caisson.

A noter que le reliquat de ce bilan énergétique non apparu dans le présent calcul, pourrait être dans la partie supérieure fluctuante du volume d'eau où les champs de vitesse n'ont pas pu être mesurés du fait de la discontinuité fréquente air-eau lors des mouvements de la surface libre.

Ce travail a été réalisé dans le cadre du Programme Européen de Recherche dans les Sciences de la Mer et la Technologie - MAST III - et plus spécialement au sein $\mathrm{du}$ groupe PROVERBS, travaillant sur les digues verticales en mer. Nous apprécions beaucoup le support financier dont nous avons bénéficier ainsi que les disdussions et commentaires pertinents du Groupe lors des ateliers et réunions.

\section{Références bibliographiques}

Allsop W., et al. The reflection of vertical walls and low reflection alternativesThird MCS Project Workshop, Delft Hydraulics, 1994

Belorgey M. Hydraulic behaviour analysis of the perforated breakwatersWorkshop on MCS structures - Hannover. Août 1992

Colmard C. Etude des phénomènes induits par les digues partielles de type caissons montés sur pieux. Thèse doct. de l'Univ.du Havre - 1996

Fugazza, Natale. Hydraulic design of perforated breakwaters-Journal of waterway. Port Coastal Ocean Eng., 118, pp1-14, 1992

Tabet Aoul et al. Etude en similitude des digues peu réfléchissantes- AUGC. Nantes-1995 - ISBN2-9509268.0.0-

Takahashi S. Design of breakwaters - Port and Harbour Research Institute February 10, 1995

Tanimoto, $\mathrm{K}$ - Yoshimoto, Y. Theoretical and experimental study of reflection coefficient for wave dissipating caisson with a permeable front wall. Proc of Coastal Structures and Breakwaters - 1995 